\title{
Alcohol dependent patients have weak negative rather than strong positive implicit alcohol associations
}

\author{
Joanne M. Dickson • Claire Gately • Matt Field
}

Received: 8 January 2013 / Accepted: 4 March 2013 /Published online: 17 March 2013

(C) The Author(s) 2013. This article is published with open access at Springerlink.com

\begin{abstract}
Rationale Alcohol dependence is characterised by motivational conflict (or ambivalence) in controlled cognitive processes, but it is unclear if ambivalence also exists within automatic cognitive processes, and if ambivalence operates between controlled and automatic processes.

Objective To investigate ambivalence operating within and between controlled and automatic processes in alcohol dependence.

Method Alcohol-dependent patients who had recently completed inpatient alcohol detoxification $(N=47)$ and social drinking controls $(N=40)$ completed unipolar implicit association tests and self-report measures of alcohol approach and avoidance motivation and alcohol outcome expectancies.

Results As predicted, both positive and negative alcohol outcome expectancies were stronger in alcohol-dependent patients compared to controls, indicative of ambivalence. Groups did not differ on implicit alcohol-positive associations, but alcohol-dependent participants had significantly weaker alcohol-negative associations than controls. Regression analyses revealed that implicit negative associations accounted for unique variance in group membership after controlling for alcohol outcome expectancies.

Conclusions Our findings demonstrate that alcohol dependent patients possess weak automatic alcohol-negative associations but not strong automatic alcohol-positive associations, and they suggest the presence of conflict between
\end{abstract}

Electronic supplementary material The online version of this article (doi:10.1007/s00213-013-3066-0) contains supplementary material, which is available to authorized users.

J. M. Dickson $\cdot$ C. Gately $\cdot$ M. Field $(\bowtie)$

School of Psychology, University of Liverpool,

Liverpool L69 7ZA, UK

e-mail: mfield@liverpool.ac.uk controlled and automatic processes with regard to negative alcohol cognitions.

Keywords Alcohol dependence · Outcome expectancies · Implicit associations · Unipolar implicit association test

Ambivalence and motivational conflict are central to addiction, including alcohol dependence (Heather 1998). Alcoholics who seek treatment report the motivation to abstain or at least reduce their consumption, yet the desire to drink remains strong (Miller 1996). It is generally accepted that approach and avoidance motivation represent two independent systems that underlie decision making processes (Carver 2001; Carver and White 1994; Eysenck 1967; Gray 1990; 2003). In alcohol dependence, inclinations to drink alcohol ('approach') and inclinations to abstain ('avoidance') represent independent motivational pathways, and their relative activation determines the decision to drink or not drink (Breiner et al. 1999). The approach and avoidance of alcohol questionnaire (AAAQ) (McEvoy et al. 2004) was developed to assess these constructs. In nondependent social drinkers, approach and avoidance inclinations are weakly negatively correlated with each other, suggesting that they are largely independent and that avoidance inclinations are not simply the inverse of approach inclinations. Among individuals with alcohol dependence, the AAAQ reveals motivational ambivalence, with alcohol dependent patients reporting stronger approach and avoidance inclinations than light social drinkers (Barkby et al. 2012; Klien et al. 2007; Stritzke et al. 2007).

Motivation is underpinned by anticipation of affective changes (Davidson et al. 2002; Gray 2003). In the context of alcohol dependence, this means that approach motivation ('want to drink') is associated with (anticipated) positive 
affective changes after drinking, and avoidance motivation ('don't want to drink') is associated with anticipated negative affective changes after drinking. Consistent with this view, the anticipated consequences of alcohol consumption underlie decisions to drink or to abstain from alcohol (Breiner et al. 1999; Cox and Klinger 1988). Alcohol outcome expectancies (AOEs) are assessed with questionnaires containing items in the format 'If I drink alcohol, then....' As would be expected, individuals who consume alcohol typically hold both positive (e.g. 'alcohol makes me more sociable') and negative (e.g. 'alcohol makes me say foolish things') AOEs. A large body of evidence demonstrates that AOEs are associated with individual differences in alcohol consumption: the strength of positive AOEs is positively correlated with quantity and frequency indices of alcohol consumption, whereas the strength of negative AOEs is negatively correlated with alcohol consumption (see (Jones et al. 2001) for a review). The relationship between AOEs and alcohol consumption appears fairly complex: positive AOEs are implicated in the initiation and establishment of alcohol involvement during adolescence, whereas negative AOEs develop after experience of the negative consequences of alcohol consumption, and then lead to reductions in alcohol consumption, in older adults (Jones and McMahon 1996; Lee et al. 1999). Individuals with alcohol dependence hold stronger positive (Brown et al. 1985; Connors et al. 1986) and negative AOEs (Li and Dingle 2012) compared to non-dependent controls, which is indicative of ambivalence in alcohol dependent patients. Moreover, both positive and negative AOEs decrease after inpatient detoxification (Spada and Wells 2008) or cognitive behaviour therapy (Young et al. 2011), and prospective studies indicate that both positive (Young et al. 2011) and negative (Jones and McMahon 1996) AOEs assessed in the clinic predict future treatment outcome.

In recent years, automatic affective alcohol associations have been studied in alcohol use disorders. Dual-process models (Wiers et al. 2007) distinguish between controlled (or explicit/reflective) and automatic (or implicit/impulsive) processes, both of which are able to influence drinking behaviour. Controlled processes are rule-based and reflective, whereas automatic processes are triggered spontaneously and without deliberation in response to a triggering stimulus (e.g. the sight of an alcoholic drink). AOEs are a prototypical example of 'controlled' alcohol-related cognitions; whereas, automatic alcohol cognitions are assessed with measures that do not rely on self-report (Roefs et al. 2011; Stacy and Wiers 2010). For example, the alcoholrelated unipolar implicit association test (IAT) (Houben and Wiers 2008a) is a speeded categorisation task in which participants must rapidly categorise words belonging to one of four categories using only two response keys. When completing the alcohol-positive IAT, participants categorise alcohol-related and positively valenced words using one key, and use a different key to categorise soda (soft-drink)related words and neutral words. On a different block of the task, participants categorise alcohol-related and neutral words using one key and soda-related words and positively valenced words using the other key. Individual differences in the speed of responding on the alcohol-positive block compared to the alcohol-neutral block are indicative of the strength of alcohol-positive associations. Previous studies that used the unipolar IAT in non-dependent drinkers revealed that alcohol-positive associations were positively correlated with alcohol consumption and problems, whereas alcohol-negative associations were unrelated to individual differences in drinking (Houben and Wiers 2006; 2008a; Jajodia and Earleywine 2003; McCarthy and Thompsen 2006). Overall, alcohol-valence associations are robust predictors of individual differences in alcohol consumption and problems within non-dependent drinkers (Stacy and Wiers 2010), and a recent meta-analysis concluded that both AOEs and implicit associations, while generally inter-correlated, explained unique variance in measures of alcohol consumption and problems (Reich et al. 2010).

Very few studies have assessed implicit alcohol-valence associations in individuals with alcohol dependence, and to our knowledge no previous study has used the unipolar IAT to investigate the strength of alcohol-positive and alcoholnegative associations (i.e. implicit 'ambivalence') in alcoholics. (De Houwer et al. 2004) administered a bipolar IAT to alcohol-dependent patients who were receiving treatment and reported that patients showed strong alcohol-negative (rather than alcohol-positive) associations. Other studies that used the bipolar IAT with non-dependent drinkers also revealed stronger alcohol-negative associations relative to alcohol-positive associations, although alcohol associations were slightly less negative in heavy drinkers compared to light drinkers (Houben and Wiers 2008b; Wiers et al. 2002). However, in these studies, the type of IAT used (bipolar IAT) means that the pattern of responding could be interpreted as strong alcohol-negative associations, weak alcohol-positive associations or a combination of the two. In the context of understanding the nature of ambivalence, it is crucial to make this distinction. Numerous studies have measured different aspects of implicit alcohol cognitions in alcohol dependent individuals (Barkby et al. 2012; Spruyt et al. 2012; Wiers et al. 2011), but importantly no previous study has measured alcohol-positive and alcohol-negative associations independently of each other or compared those indices in alcoholdependent patients with a non-dependent control group.

Our goal in the present study was to characterise ambivalence in alcohol-dependent patients in comparison with a control group of social drinkers. We predicted that relative to controls, alcohol dependent individuals would (1) report stronger positive and negative alcohol outcome expectancies and (2) exhibit stronger positive and negative implicit 
alcohol associations on the IAT. The latter hypothesis has never been investigated, and in order to extend a recent meta-analysis (Reich et al. 2010) we conducted additional analyses to investigate whether implicit alcohol associations would explain unique variance in group membership (alcohol-dependent vs. control), after controlling for the self-report measures.

\section{Method}

\section{Participants}

Alcohol-dependent participants Forty-seven participants (29 male, 18 female; $M=43.57$ years, $S D=9.97$ ) were recruited from a specialist alcohol treatment unit in NW England. Prior to detoxification, structured clinical interviews were conducted by trained clinicians to determine the diagnosis of alcohol dependence according to ICD-10 criteria. Participants had received medical detoxification on an inpatient basis before remaining on the ward for a 5-day group relapse prevention programme (based on cognitive behavioural therapy principles) before discharge. Eligible participants were invited to participate towards the end of the relapse prevention programme, when they were no longer experiencing symptoms of alcohol withdrawal and were fit for discharge. From a potential pool of 56 participants, 47 respondents consented to take part in the study ( $84 \%$ ).

Social drinker (control) participants Forty (17 Male, 23 Female, $M=36.48$ years, $S D=13.23$ ) light social drinkers were recruited from the same region in NW England and from a range of community organisations. Exclusion criteria for the non-clinical group were weekly alcohol consumption at levels above those recommended by the UK government national guidelines ( 21 and 14 units ( 1 unit $=8 \mathrm{~g}$ alcohol) per week for males and females, respectively). In addition, participants were excluded if they reported a history of alcohol dependence.

Exclusion criteria for both groups included (1) current dependence on other substances (apart from nicotine), (2) medical illness, (3) positive breath alcohol level, (4) psychosis and (5) overt cognitive impairment. All participants spoke fluent English and had normal or corrected-to-normal vision.

Materials and measures

Time line follow back (Sobell et al. 1979) Participants recorded their alcohol consumption over the previous week in this retrospective diary, which allowed us to calculate the number of standard units of alcohol ( 1 unit $=8 \mathrm{~g}$ alcohol) they had consumed in the previous week.
Leeds dependence questionnaire (Raistrick et al. 1994) The 10-item Leeds dependence questionnaire was used to assess severity of alcohol dependence.

The hospital anxiety and depression scales (Zigmond and Snaith 1983). The 14-item hospital anxiety and depression scales (HADS) was used to assess state anxiety and depression.

Approach and avoidance of alcohol questionnaire (McEvoy et al. 2004). The 20-item questionnaire was used to assess approach and avoidance motivation in relation to drinking. (see Supplementary materials for details).

Comprehensive effects of alcohol questionnaire (Fromme et al. 1993). This 38-item questionnaire yields values for four positive outcome expectancies (sociability, tension reduction, liquid courage, sexuality) and three negative outcome expectancies (cognitive and behavioural impairment, risk and aggression, self-perception). We computed a mean value for positive AOEs and a mean value for negative AOEs. In the present study, internal consistency for the subscales was acceptable for the alcohol dependent group $(\alpha=.70$ to $\alpha=.89$ ) and control group ( $\alpha=.76$ to $\alpha=.85$ ).

Unipolar implicit association tests (Houben and Wiers 2008a) We used two separate unipolar IATs: a positiveneutral version and a negative-neutral version. In the positive-neutral IAT, participants categorised words belonging to four categories (positive, neutral, alcohol-related, soft drink-related). In one block of the positive-neutral IAT, participants pressed either the left (or the right) key to categorise alcohol-related and positive words and the right (or the left) key to categorise soft drink-related and neutral words. In a different block of the task, participants pressed the left (or right) key to categorise alcohol-related and neutral words and the right (or left) key to categorise soft drink-related and positive words. The negative-neutral IAT was similar, although negative words were used in place of positive words. Each IAT comprised of five sub-blocks, each comprising 20 trials.

In the first block (20 trials), participants categorised only alcohol-related and soft drink-related words. In the second block (20 trials), participants categorised only positive (or negative) and neutral words. The third block (40 trials) was the first block of critical trials, and in this block all four categories of words were presented. In the fourth block (20 trials), the mapping of positive (or negative) and neutral words to response keys was reversed, and again participants categorised only positive (or negative) and neutral words. The fifth block (40 trials) was the second block of critical trials, and in this block all four categories of words were 
again presented. The critical contrast is between the third and fifth blocks of the task. For example, if participants are faster to respond when alcohol words and positive words share a response key compared to when alcohol words and neutral words share a response key, this indicates that alcohol-positive associations are stronger than alcoholneutral associations.

On each trial, the target words were presented in white text against a black background. Category labels (e.g. 'alcohol or positive' and 'soft-drink or neutral') were presented in the top left and right hand sides of the screen, corresponding to the location of response keys on that block. Target stimuli remained on screen until participants made a response or until a $3-\mathrm{sec}$ timeout had elapsed. If participants made an error, or did not respond before the timeout period, feedback ('wrong!') was presented in red text below the target stimuli for $300 \mathrm{~ms}$. No feedback was given for correct responses. The inter-trial interval was $500 \mathrm{~ms}$. The following variables were randomised across participants: order of completion of IATs (positive-neutral first vs. negative-neutral first); order of completion of task blocks (alcohol-positive or alcohol-negative first vs. alcohol-neutral first) and key assignment for alcohol and soft-drink categories (alcohol responses on left or on right).

\section{Preliminary statistical analysis}

The strength of positive and negative alcohol associations was calculated using the ' $d$ ' measure (Greenwald et al. 2003). This algorithm is recommended when there are likely to be between-group differences in baseline speed of responding (as was the case here, the alcohol-dependent group were significantly slower overall than the control group), and it also tends to improve correlations between automatic and self-report measures. Full details are available on request, but we note here that the primary results were unaffected when raw reaction times were used in the analyses

\section{Procedure}

The study was approved by the University and the Local Research Ethics Committee and NHS Trust Research Governance Committee. Informed consent was obtained prior to testing. Alcohol dependent participants were tested individually in private rooms in the clinic, and the control participants were tested in private rooms in a range of locations (e.g. community centres). Testing sessions lasted approximately 30-45 min. All participants completed the IATs followed by the comprehensive effects of alcohol questionnaire (CEOA) and other self-report measures.

\section{Results}

\section{Group characteristics}

Table 1 presents summary statistics for drinking characteristics and HADS scores for the alcohol dependent and control groups.

Self-reported alcohol-related outcome expectancies (CEOA)

CEOA scores were analysed using a mixed ANOVA comprising a within-subjects factor, expectancy valence (positive vs. negative) and between-subjects factors of group (alcohol dependent vs. control) and gender. Given that the alcohol-dependent participants were significantly older than controls $(t(85)=2.85, p<.01)$, age was added as a covariate. Results revealed a significant main effect of group, $F(1,82)=29.86, p<.001, \eta_{p}^{2}=.27$, which was qualified by a group by expectancy valence interaction, $F(1,82)=8.97, p<.001, \eta_{p}^{2}=.10$. There were no other significant main effects or interactions, $p>.2$. To decompose the significant group by expectancy valence interaction, we first compared groups on positive AOEs and negative AOEs, separately. As predicted, alcohol dependent participants reported stronger negative AOEs, $t(85)=6.13, p<.001$ and stronger positive AOEs, $t(85)=2.83, p<.01$, than controls, as can be seen in Table 2 . The group $\times$ expectancy valence interaction reflects the observation that negative AOEs tended to be stronger than positive AOEs in the alcohol dependent group, $t(46)=1.75, p=0.09$, whereas the control group showed the opposite pattern, $t(39)=3.52, p<.01$.

\section{Automatic alcohol associations}

Association strength was analysed using a mixed ANOVA comprising a within-subjects factor, alcohol association valence (positive vs. negative), between-subjects factors of Group (alcohol dependent vs. control) and gender, and age as a covariate. Results revealed a significant main effect of alcohol association valeence, $F(1,82)=5.09, p<.05$, $\eta^{2}=.06$, and group $F(1,82)=12.25, p<.001, \eta^{2}=.13$, which was qualified by a significant group by alcohol association valence interaction, $F(1,82)=6.08, p<.05, \eta^{2}=.07$. There were no other significant main effects or interactions $(p>.07)$. Between-group contrasts revealed that groups did not differ on automatic positive alcohol associations, $t(85)=.34, p=.34$. However, contrary to predictions, the alcohol dependent group had significantly weaker automatic negative alcohol associations than controls, $t(85)=4.30, p<.001)$. Within-subject contrasts revealed that automatic negative alcohol associations tended to be stronger than automatic positive alcohol associations in both groups, although this difference was more robust in the 
Table 1 Alcohol dependent and control group means (SD) for drinking characteristics and mood

\begin{tabular}{|c|c|c|c|c|c|c|}
\hline \multirow[b]{2}{*}{ Age start drinking } & \multicolumn{2}{|c|}{ Alcohol dependent $(n=47)$ mean $(\mathrm{SD})$} & \multicolumn{2}{|c|}{ Control $(n=40)$ mean (SD) } & \multirow{2}{*}{$\begin{array}{l}t \text { value } \\
<1\end{array}$} & \multirow[t]{2}{*}{$\mathrm{M}-\mathrm{W} \mathrm{U}$} \\
\hline & 16.28 & $(3.55)$ & 16.73 & $(1.59)$ & & \\
\hline Weekly drinking days & 6.89 & $(0.60)$ & 1.98 & $(1.48)$ & & $-8.41^{*}$ \\
\hline Total weekly units & 303.75 & $(159.45)$ & 7.46 & $(6.47)$ & & $-8.00^{*}$ \\
\hline Total LDQ score & 24.15 & $(5.30)$ & 0.53 & $(0.78)$ & $33.46^{*}$ & \\
\hline HADS anxiety & 11.19 & $(4.95)$ & 4.30 & $(2.70)$ & $7.86^{*}$ & \\
\hline HADS depression & 7.74 & $(4.79)$ & 1.55 & $(1.92)$ & $7.66^{*}$ & \\
\hline
\end{tabular}

$* * * p<.001$

control group, $t(39)=4.67, p<.001$, than in the alcohol dependent group, $t(46)=1.91, p=.06$.

We note that for the analyses of both alcohol outcome expectancies and automatic associations, the group differences reported above remained significant when we did not incorporate age and gender in the analyses.

Correlations between outcome expectancies and automatic associations

In the alcohol-dependent group, positive expectancies and alcohol-positive associations were not significantly correlated, $(r=-.04, p=.78)$ and neither were negative expectancies and alcohol-negative associations $(r=-.01, p=.96)$. In the control group, there was a significant positive correlation between positive expectancies and alcohol-positive associations $(r=.42, p=007)$, but negative expectancies and alcohol-negative associations were not significantly correlated $(r=.04, p=.79)$.

Self-reported expectancies and automatic associations as predictors of group membership

To investigate the relative contributions of alcohol outcome expectancies and automatic alcohol associations as predictors of group membership (alcohol dependent vs. control), we used a logistic regression. Gender and age were entered in the first step, which was significant $\left(\chi^{2}(2)=10.58, p=.005\right)$.

Table 2 Group means (SDs) on alcohol outcome expectancies and automatic alcohol associations

\begin{tabular}{lll}
\hline & Alcohol dependent patients & Controls \\
\hline CEOA positive & $2.76(0.60)$ & $2.41(0.53)$ \\
CEOA negative & $2.97(0.72)$ & $2.10(0.58)$ \\
IAT positive alcohol $(d)$ & $.03(.26)$ & $.11(.48)$ \\
IAT negative alcohol $(d)$ & $.16(.43)$ & $.63(.58)$ \\
\hline
\end{tabular}

CEOA positive $=$ positive expectancy, $\mathrm{CEOA}$ negative $=$ negative $\mathrm{ex}-$ pectancy, IAT positive alcohol=automatic positive alcohol associations, IAT negative alcohol $=$ automatic negative alcohol associations
Age was a significant predictor $($ Wald $=6.27, p=.01)$, but gender was not $($ Wald $=1.01, p=.31)$. Self-reported positive and negative alcohol outcome expectancies were entered simultaneously in the second step, and positive and negative alcohol associations (from the IAT) were entered in the third step. Results for each model are presented in Table 3. In the second step, the overall model was significant $\left(\chi^{2}(4)=63.98, p<.001\right)$. Positive alcohol expectancies (Wald $=7.03, p=008)$ and negative alcohol expectancies (Wald $=16.78, p<.001$ ) each predicted group membership. In the third step, positive alcohol expectancies ( Wald $=5.60$, $p=.02$ ) and negative alcohol expectancies (Wald $=10.48$, $p=.001$ ) remained significant predictors, and the inclusion of the automatic alcohol associations significantly improved the model fit $\chi^{2}(6)=78.56, p<.001$. Results showed that automatic negative alcohol associations significantly predicted group membership ( Wald $=7.38, p=.007$ ) but automatic positive alcohol associations did not ( Wald $=0.39, p=.53)$. In summary, the final model distinguished alcohol dependent participants from light social drinkers by stronger positive and negative AOEs and weaker automatic negative alcohol associations, after controlling for participants' age and gender. The final model correctly classified $90.1 \%$ of participants' group membership.

\section{Discussion}

We investigated ambivalence within and between selfreported and automatic alcohol-related cognitions in alcohol dependent patients and a control group of social drinkers. As predicted, alcohol-dependent participants reported stronger positive and negative AOEs than the control group. Contrary to our hypotheses, alcohol dependent participants showed weaker automatic negative alcohol associations than social drinkers, but groups did not differ significantly on automatic positive alcohol associations. Further, regression analysis revealed that weak automatic negative alcohol associations and strong self-reported negative and positive AOEs each significantly distinguished alcohol dependent participants from light social drinkers. 
Table 3 Logistic regression showing predictors of group membership (alcohol dependent vs. control)

\begin{tabular}{|c|c|c|c|c|c|c|}
\hline \multirow[b]{2}{*}{ Model } & \multirow[b]{2}{*}{ Predictor } & \multirow[b]{2}{*}{$B$} & \multirow[b]{2}{*}{ SE $B$} & \multicolumn{3}{|c|}{$95 \% \mathrm{CI}$ for exp $\mathrm{b}$} \\
\hline & & & & Lower & $\operatorname{Exp} b$ & Upper \\
\hline \multirow[t]{2}{*}{1} & Gender & -.49 & .49 & & & \\
\hline & Age & $.05^{*}$ & .02 & 1.01 & 1.06 & 1.10 \\
\hline \multirow[t]{4}{*}{2} & Gender & -.49 & .76 & & & \\
\hline & Age & $.11^{*}$ & .05 & 1.02 & 1.12 & 1.22 \\
\hline & CEOA pos & $2.62 * *$ & .99 & 1.98 & 13.80 & 96.03 \\
\hline & CEOA neg & $3.51 * * *$ & .86 & 6.23 & 33.35 & 178.59 \\
\hline \multirow[t]{6}{*}{3} & Gender & -1.01 & 1.06 & 0.97 & 1.08 & 1.19 \\
\hline & Age & .08 & .05 & & & \\
\hline & CEOA pos & $3.30 *$ & 1.39 & 1.76 & 27.20 & 419.31 \\
\hline & CEOA neg & $5.27 * *$ & 1.63 & 8.01 & 195.06 & 4749.60 \\
\hline & IAT pos alcohol $(d)$ & -.73 & 1.17 & 05 & 0.48 & 4.79 \\
\hline & IAT neg alcohol $(d)$ & $-3.97 * *$ & 1.46 & 0.001 & 0.19 & 0.33 \\
\hline
\end{tabular}

$N=81$. Six cases (two control and four alcohol-dependent participants) were removed due to extreme residual $z$-scores $> \pm 2.8$

Model 1: Cox \& Snell $R^{2}=.12$, Nagelkerke $R^{2}=.16$, Hosmer \& Lemeshow $\chi^{2}(8)=4.54, p=.81$

Model 2: Cox \& Snell $R^{2}=.55$, Nagelkerke $R^{2}=.73$, Hosmer and Lemeshow $\chi^{2}(8)=10.22, p=.25$

Model 3: Cox \& Snell $R^{2}=.62$, Nagelkerke $R^{2}=.83$, Hosmer \& Lemeshow $\chi^{2}(8)=4.73, p=.79$

$* p<.05 ; * * p \leq .01 ; * * * p<.001$

The primary novel finding in our study came from the unipolar implicit association test, and we highlight that ours is the first study to administer this task to alcohol-dependent patients. We found group differences in the strength of automatic negative alcohol associations, but these differences were not in the direction that we originally hypothesised. Alcohol dependent and control groups did not differ on the strength of positive associations, although the alcohol dependent group showed weaker automatic negative associations on the IAT compared to the control group. Our findings contrast with a study by (De Houwer et al. 2004), in which a bipolar IAT was used. In that study, alcohol dependent patients receiving treatment were characterised by strong alcoholnegative associations, but importantly this was relative to alcohol-positive associations, because positive and negative associations could not be distinguished with the bipolar IAT. Previous studies that used the bipolar IAT have also characterised social drinkers as having stronger alcoholnegative associations than alcohol-positive associations (Houben and Wiers 2008b; Wiers et al. 2002). In the present study, both groups displayed this profile of automatic alcohol associations (in that alcohol-negative associations were stronger than alcohol-positive associations), although this within-subject difference was more robust in social drinkers than in the alcohol-dependent group. One explanation for our findings is that strong automatic negative alcohol associations ordinarily develop as a consequence of the negative consequences of drinking (e.g. hangover), or they may reflect internalisation of negative societal attitudes toward alcohol (Stacy and Wiers 2010). Once automatic negative alcohol associations are established, they may act as a protective 'brake' on drinking behaviour in nondependent drinkers. If automatic negative associations fail to develop, this may confer an increased risk of developing alcohol dependence. Prospective studies with participants in the early (e.g. adolescents) and latter (e.g. older adults with alcohol dependence) stages of alcohol involvement are required to investigate this issue. For example, weak alcohol-negative associations may predict the risk of relapse to heavy drinking in alcohol-dependent patients.

Consistent with recent findings, ( $\mathrm{Li}$ and Dingle 2012) alcohol dependent participants were characterised by stronger positive and negative AOEs than the control group. Selfreported positive and negative AOEs and automatic negative alcohol associations each significantly predicted group membership, but automatic positive alcohol associations did not. After controlling for self-reported AOEs, weak automatic negative alcohol associations accounted for additional variance when distinguishing alcohol-dependent individuals from light social drinkers. Our findings are consistent with a meta-analysis (Reich et al. 2010), which demonstrated that self-reported AOEs and implicit alcohol associations each explained unique variance in measures of alcohol consumption and problems, although self-report measures explained the majority of variance. What is most notable about our regression results is that group membership was best predicted by the combination of strong self-reported positive and negative AOEs and weak automatic negative alcohol associations, 
which suggests that this conflict or discrepancy between controlled and automatic processes is an important feature of alcohol dependence. Research in other domains of psychology has highlighted the importance of studying the discrepancy between controlled (or explicit) and automatic (or implicit) cognitive processes (Brinol et al. 2006). Our results show that this discrepancy also exists in alcohol-dependent patients, and further work is required to investigate the clinical significance of this discrepancy. For example, does the magnitude of the implicit-expectancy discrepancy predict relapse to heavy drinking following treatment, and could resolution of this discrepancy improve the outcome of treatment?

A few final observations deserve comment. First, all the alcohol-dependent participants were tested shortly after they had completed an inpatient detoxification programme and were soon to be discharged. We note that participants completed the study procedures when they were no longer experiencing acute withdrawal symptoms, and there was very little variation in the duration of abstinence from alcohol in this sample. For this reason, it is unclear if the observed characteristics of our alcohol-dependent group are a relatively stable feature of alcohol dependence or a more transient characteristic of alcoholics who have recently completed detoxification. Future studies are required to investigate the role of the duration of alcohol abstinence on self-reported and automatic alcohol cognitions, and to characterise alcohol cognitions in alcohol-dependent individuals who have not yet attempted to abstain from, or reduce, their alcohol consumption. Second, our regression findings point to a discrepancy between automatic and controlled alcohol-related cognitions in alcohol-dependent participants. However, it should be noted that the IAT involves a contrast between soft drink and alcohol-related stimuli, whereas the outcome expectancy measure (CEOA) only assesses outcome expectancies for alcoholic drinks. In order to make direct comparisons between the two measures, future studies could contrast alcohol outcome expectancies with outcome expectancies for soft drinks, an issue that has not been previously investigated (to our knowledge).

Finally, polysubstance abusers with alcoholism have difficulty switching from one set of task rules to another when performing cognitive tasks (Noel et al. 2005). Given this, it is possible that the alcohol dependent patients in our study may have had problems with mental flexibility and perseveration, which could have impaired their ability to switch between blocks in the IAT (e.g. switching from the 'alcohol+negative' block to the 'alcohol+neutral' block). However, a general deficit in mental flexibility would be expected to inflate the magnitude of effects on the IAT, which does not seem to have occurred in our study because patients had weaker rather than stronger alcohol-negative associations compared to social drinker controls.
To conclude, our results revealed that compared to controls, alcohol dependent individuals undergoing treatment reported stronger positive and negative alcohol outcome expectancies, indicative of cognitive ambivalence. Counter to expectations, negative automatic alcohol associations were weaker in alcohol dependent individuals compared to social drinkers, but groups did not differ on the strength of automatic positive alcohol associations. This is the first study to use the unipolar IAT to investigate the strength of alcohol-positive and alcohol-negative associations in inpatient alcoholics. Notably, the contrasting pattern of negative alcohol cognitions in controlled versus automatic processes highlights the importance of automatic processes, particularly when they conflict with controlled processes, in alcohol dependence.

Acknowledgments This research was funded by a grant from the Wellcome Trust, reference 068247/Z/08/Z. Authors have no conflicts of interests.

Open Access This article is distributed under the terms of the Creative Commons Attribution License which permits any use, distribution, and reproduction in any medium, provided the original author(s) and the source are credited.

\section{References}

Barkby H, Dickson JM, Roper L, Field M (2012) To approach or avoid alcohol? Automatic and self-reported motivational tendencies in alcohol-dependence. Alcohol Clin Exp Res 36:361-368

Breiner MJ, Stritzke WG, Lang AR (1999) Approaching avoidance: A step essential to the understanding of craving. Alcohol Research and Health 23:197-206

Brinol P, Petty RE, Wheeler SC (2006) Discrepaancies between explicit and implicit self-concepts: consequences for information processing. J Pers Soc Psychol 91:154-170

Brown SA, Goldman MS, Christiansen BA (1985) Do alcohol expectancies mediate drinking patterns of adults? J Consult Clin Psychol 53:512-519

Carver CS (2001) Affect and the functional bases of behaviour: on the dimensional structure of affective experience. Personality and Social Psychology Review 5:345-356

Carver CS, White TL (1994) Behavioural inhibition, behavioural activation, and affective responses to impending reward and punishment: the BIS/BAS scales. J Personal Soc Psychol 67:319-333

Connors GJ, O'Farrell TJ, Cutter HS, Thompson DL (1986) Alcohol expectancies among male alcoholics, problem drinkers, and nonproblem drinkers. Alcohol Clin Exp Res 10:667-671

Cox WM, Klinger E (1988) A motivational model of alcohol use. J Abnorm Psychol 97:168-180

Davidson RJ, Pizzagalli D, Nitschke JB, Putnam KM (2002) Depression: perspectives from affective neuroscience. Annu Rev Psychol 53:545-574

De Houwer J, Crombez G, Koster EHW, Beul ND (2004) Implicit alcohol-related cognitions in a clinical sample of heavy drinkers. Journal of Behaviour Therapy and Experimental Psychiatry $35: 275-286$

Eysenck H (1967) The biological basis of personality. Thomas, Springfield, IL 
Fromme K, Stroot EA, Kaplan D (1993) Comprehensive effects of alcohol: development and psychometric assessment of a new expectancy questionnaire. Psychol Assess 5:19-26

Gray JA (1990) Brain systems that mediate both emotion and cognition. Cognition and Emotion 4:269-288

Gray JA (2003) The neuropsychology of anxiety: An enquiry into the functions of the septo-hippocampal system. Oxford University Press, New York

Greenwald AG, Nosek BA, Nanaji MR (2003) Understanding and using the Implicit Association Test: I. An improved scoring algorithm Journal of Personality and Social Psychology 85:197-216

Heather N (1998) A conceptual framework for explaining drug addiction. J Psychopharmacol 12:3-7

Houben K, Wiers RW (2006) A test of the salience asymmetry interpretation of the alcohol-IAT. Exp Psychol 53:292-300

Houben K, Wiers RW (2008a) Implicitly positive about alcohol? Implicit positive associations predict drinking behaviour. Addict Behav 33:979-986

Houben K, Wiers RW (2008b) Measuring implicit alcohol associations via the Internet: Validation of Web-based implicit association tests. Behavior Research Methods 40:1134-1143

Jajodia A, Earleywine M (2003) Measuring alcohol expectancies with the implicit association test. Psychol Addict Behav 17:126-133

Jones B, Corbin WR, Fromme K (2001) A review of expectancy theory and alcohol consumption. Addiction 96:57-72

Jones B, McMahon J (1996) A comparison of positive and negative alcohol expectancy and value and their multiplicative composite as predictors of post-treatment abstinence survivorship. Addiction 91:89-99

Klien AA, Stasiewicz PR, Koutsky JR, Bradizza CM, Coffey SE (2007) A psychometric evaluation of the approach and avoidance alcohol questionnaire (AAAQ) in alcohol dependent outpatients. J Psychopathol Behav Assess 29:231-240

Lee NK, Greely J, Oei TPS (1999) The relationship of positive and negative alcohol expectancies to patterns of consumption of alcohol in social drinkers. Addict Behav 24:359-369

Li HK, Dingle GA (2012) Using the Drinking Expectancy Questionnaire (revised scoring method) in clinical practice. Addict Behav 37:198-204

McCarthy DM, Thompsen DM (2006) Implicit and explicit measures of alcohol and smoking cognitions. Psychol Addict Behav 20:436-444

McEvoy PM, Stritzke WG, French DJ, Lang AR, Ketterman R (2004) Comparison of three models of alcohol craving in young adults: a cross validation. Addiction 99:482-497

Miller WR (1996) Motivational interviewing: research, practice, and puzzles. Addict Behav 21:835-842

Noel X, Van der Linden M, D'Acremont M, Colmant M, Hanak C, Pelc I, Verbanck P, Bechara A (2005) Cognitive biases toward alcohol-related words and executive deficits in polysubstance abusers with alcoholism. Addiction 100:1302-1309

Raistrick D, Bradshaw J, Tober G, Weiner J, Allison J, Healey C (1994) Development of the Leeds dependence questionnaire (LDQ): a questionnaire to measure alcohol and opiate dependence in the context of a treatment evaluation package. Addiction $89: 563-572$

Reich RR, Below MC, Goldman MS (2010) Explicit and implicit measures of expectancy and related alcohol cognitions: a meta-analytic comparison. Psychol Addict Behav 24:13-25

Roefs A, Huijding J, Smulders FTY, MacLeod CM, de Jong PJ, Wiers RW, Jansen ATM (2011) Implicit measures of association in psychopathology research. Psychol Bull 137:149-193

Sobell LC, Maisto SA, Sobell MB, Cooper AM (1979) Reliability of alcohol abusers' self-reports of drinking behaviour. Behav Res Ther 17:157-160

Spada MM, Wells A (2008) Metacognitive beliefs about alcohol use: development and validation of two self-report scales. Addict Behav 33:515-27

Spruyt A, De Houwer J, Tibboel H, Vershuere B, Crombez G, Verbanck P, Brevers D, Noel X (2013) On the predictive validity of automatically activated approach/avoidance tendencies in abstaining alcohol-dependent patients. Drug Alcohol Depend 127(1-3):81-6

Stacy AW, Wiers RW (2010) Implicit cognition and addiction: a tool for explaining paradoxical behaviour. Annu Rev Clin Psychol 6:551-575

Stritzke WG, McEvoy PM, Wheat LR, Dyer KR, French DJ (2007) The yin and yang of indulgence and restraint: the ambivalence model of craving. In: O'Neal PW (ed) Motivation of Health Behaviour. Nova Science, Huntingdon, New York

Wiers RW, Bartholow BD, van den Wild E, Thush C, Engels RC, Sher KJ, Grenard J, Ames SL, Stacy AW (2007) Automatic and controlled processes and the development of addictive behaviours in adolescents: a review and a model. Pharmacol Biochem Behav $86: 263-283$

Wiers RW, Eberl C, Rinck M, Becker ES, Lindenmeyer J (2011) Retraining automatic action tendencies changes alcoholic patients' approach bias for alcohol and improves treatment outcome. Psychol Sci 22:490-497

Wiers RW, Van Woerden N, Smulders FTY, De Jong PJ (2002) Implicit and explicit alcohol-related cognitions in heavy and light drinkers. J Abnorm Psychol 111:648-658

Young RM, Connor JP, Feeney GFX (2011) Alcohol expectancy changes over a 12 -week cognitive-behavioural therapy programme are predictive of treatment success. J Subst Abus Treat 40:18-25

Zigmond AS, Snaith RP (1983) The Hospital Anxiety and Depression Scale. Acta Psychiatr Scand 67:361-370 